\title{
INTELIGENCIA TERRITORIAL: CONCEPTUALIZACIÓN Y AVANCE EN EL ESTADO DE LA CUESTIÓN. VÍNCULOS POSIBLES CON LOS DESTINOS TURÍSTICOS
}

\author{
María Jesús Perea-Medina \\ Enrique Navarro-Jurado \\ Ana María Luque-Gil
}

Universidad de Málaga

\section{RESUMEN}

En el documento se presenta la definición, evolución y elementos clave del concepto inteligencia territorial (IT). Así, se muestran las herramientas desarrolladas desde la IT y un estudio de las aplicaciones llevadas a cabo, teniendo en cuenta las herramientas, ubicación y temática. Por el objeto de esta investigación, donde se trata de vincular IT y turismo, se muestran relevantes los casos donde se han aplicado ambos conceptos. La búsqueda de experiencias en turismo hace imprescindible una valorización de las comunidades anfitrionas, y esto es posible a través de la IT.

Palabras clave: inteligencia territorial; desarrollo sostenible; gobernanza; TIC; turismo.

Territorial Intelligence: conceptualization and progress in the status of the issue. Possible links to tourist destination.

\section{ABSTRACT}

The document presents the definition, evolution and key elements of Territorial Intelligence (TI). Subsequently, shown the tools developed from the TI and a study of the applications carried out, taking into account the implemented tools, location and subject. The aim of this

Recibido: 24 de marzo de 2017

Devuelto para su revisión: 13 de junio de 2017

Aceptado: 26 de julio de 2017

Facultad de Turismo. Universidad de Málaga. Calle de León Tolstoi s/n. 29071 MÁLAGA (España). E-mail: maria_vva_vva@hotmail.com, enavarro@uma.es,geoana@uma.es 
research, which it's generate a link between TI and tourism, are relevant cases where both concepts has been applied. Experiences search raises a new way of tourism, so is essential that tourism find a valorization of host communities, and this is possible through the TI.

Keyword: territorial intelligence; sustainable development; governance; ICT; tourism.

\section{INTRODUCCIÓN}

Algunos de los problemas que la sociedad experimenta en la actualidad se deben al modelo económico imperante, basado en el consumo, donde el crecimiento ilimitado es la máxima que canibaliza los recursos naturales y culturales, y conlleva desigualdades sociales cada vez más pronunciadas. Una sociedad que busque solucionar estas diferencias por medio de medidas no sólo económicas sino integrales, debe actuar con inteligencia dado el grado de conocimiento existente. Es en este contexto en el que surge el concepto de "inteligencia territorial", con los tres elementos que la definen: desarrollo sostenible, gobernanza y Nuevas Tecnologías de la Información y la Comunicación.

En otro orden de cosas, una de las actividades de mayor importancia global es el turismo, que se define bajo reglas de consumo de tiempo de ocio, y donde la mercantilización de los recursos, estandarización y homogeneización han marcado su evolución en el tiempo. A día de hoy, existen nuevas formas de hacer turismo, donde prima lo auténtico y la búsqueda de experiencias, de ahí que el patrimonio, la cultura, la identidad local, se hayan convertido en aspectos fundamentales por los que apostar los gestores de los destinos turísticos. El turismo es un sector que se desarrolla en un territorio en concreto, que es capaz de generar experiencias a través de sus recursos materiales e inmateriales, y donde existen una gran diversidad de actores involucrados, por tanto, a priori podría ser partícipe de iniciativas propias de la inteligencia territorial.

Con estos dos elementos como base -inteligencia territorial y turismo- el objetivo básico de la comunicación es realizar una primera aproximación entre al vínculo referido, atendiendo a aspectos conceptuales, analizando aplicaciones-proyectos desarrollados e investigando los puntos de conexión entre ambos términos. Para ello, la comunicación comienza definiendo el concepto y evolución del término inteligencia territorial, continua explicando los elementos clave, sigue con la presentación de determinadas herramientas y aplicaciones que se han llevado a cabo, para concluir, con ejemplos concretos que vinculan la inteligencia territorial y el turismo. Por último, a modo de reflexión, se plantean algunas líneas de investigación futuras que pueden ser de utilidad en el avance de estos planteamientos novedosos en el marco del uso turístico de los territorios. Es necesario plantear que a día de hoy existen muy pocos trabajos donde se vincule inteligencia territorial y turismo, mientras que en otros campos de actuación, como el empleo, la planificación territorial, la integración social o la pobreza, si son más abundantes. Es por esto, que la presente comunicación trata de aportar el estado de la cuestión, con el fin de poder avanzar en la vinculación entre inteligencia territorial y turismo, elaborando una propuesta de tesis doctoral que vincule ambos conceptos y experimentarlo en Málaga. 


\section{CONCEPCIÓN Y EVOLUCIÓN DEL TÉRMINO INTELIGENCIA TERRITO- RIAL}

El concepto y las herramientas propias de la inteligencia territorial tienen su origen a finales de los años ochenta, para dar respuesta a las necesidades de un grupo de actores territoriales que trabajaban en proyectos de lucha contra la pobreza (Miedes et al., 2010). En 1989, la ausencia de planes territoriales basados en la participación, la exigencia de planteamientos críticos en el ámbito del desarrollo sostenible, y la revolución de las tecnologías de la información y la comunicación (TICs), dan lugar a que surja en Europa (Besançon, Francia) el método "Catalyse" (Girardot, 2010), cuyo principal objetivo era la creación de inteligencias colectivas a través de diversas herramientas (de diagnóstico y de evaluación), con el fin de que los actores pudiesen elaborar, argumentar, animar y evaluar proyectos de desarrollo sostenible. Desde Francia y a partir de 1994, este proyecto se extendió a España, Bélgica, Rumanía y Hungría. No obstante, hay que esperar al año 2000 para que se plantee la primera definición de Inteligencia Territorial, entendida como "un medio para los investigadores, para los actores y para la comunidad territorial de adquirir un mejor conocimiento del territorio, pero también de controlar mejor su desarrollo (...)" (Girardot, 2010: 15). Esta tesis inicial ya recoge los dos aspectos fundamentales que marcarán otras definiciones: el enfoque científico y los actores-comunidad territorial (Girardot, 2002; Bertacchini, 2004; Devillet et al., 2008; Girardot, 2008; Bozzano, 2010; Almansa, 2010; Miedes et al., 2010; Frediani, 2012; Poujol, 2013; Sánchez, 20132; Gliemmo, 2013; Marek, 2013³). El enfoque científico en la inteligencia territorial tiene como función integrar y desarrollar el conocimiento multidisciplinario y los métodos necesarios para comprender las estructuras territoriales, los sistemas territoriales y las dinámicas de los territorios (Soulier et al., 2011). De ello se deduce que la inteligencia territorial no puede ser vista como una disciplina, no es la simple suma de conocimientos, sino que se conforma como un conjunto de conocimientos obtenidos mediante la observación y el razonamiento estructurado.

Los actores-comunidad territorial suponen el segundo pilar de la inteligencia territorial, de hecho ésta se define como un proceso que se basa en el entendimiento que articula el conocimiento, siendo imprescindibles la co-participación, la co-construcción y la colaboración llevada a cabo por los diferentes actores (Bozzano, 2010; Frediani, 2012; Masselot, 2008). Con estos tres ítems, se puede trabajar para generar el desarrollo integral de las zonas con mayores dificultades, lo que se traduce en una mejor articulación y combinación de los objetivos económicos, sociales y ambientales de un territorio. De hecho, Bozzano (2011) argumenta que un territorio nunca podrá ser inteligente sin la presencia de los actores que son los que le imprimen dicha cualidad (metáfora de la mesa) ${ }^{4}$. Así pues,

1 Poujol, L. citado en Bozzano (2013).

2 Sánchez, C. citado en Bozzano (2013).

3 Marek, A. citado en Seminario en Minas (2013).

4 La metáfora de la mesa plantea de manera muy representativa como se trabaja con la inteligencia territorial, y hace referencia a la co-construcción de transformaciones con actores territoriales. Existen cuatro patas compuestas por los actores políticos, sociales, económicos y científicos. La tabla de la mesa representa el territorio por transformar. Encima de la mesa hay tartas, que simulan los proyectos que eligen los actores, y cada una 
se habla de inteligencia territorial cuando los actores -gestores o no- conocen bien el territorio o se esfuerzan para conocerlo a través del acceso a información de todo tipo, siempre y cuando dicho conocimiento se utilice, y esto es importante recordarlo, de forma sostenible territorialmente.

Junto al enfoque científico y los actores, las herramientas se convierten en piezas clave de todo este proceso, fundamentalmente porque la actual sociedad de la información cuenta con métodos científicos y herramientas que, a través de las TICs, pueden llegar a los actores que lo deseen y facilitar la búsqueda de alternativas al despilfarro de recursos. De hecho, en la anterior definición se reconoce que “(...) la apropiación de las tecnologías de la comunicación y de la información, en sí misma, es una etapa indispensable para que los actores introduzcan un proceso de aprendizaje que les permitirá obrar de manera pertinente y eficiente (...)" (Girardot, 2010: 15). Es por ello que el concepto "información" ha de entenderse desde el intercambio, la mutualización, la organización y el conocimiento, para así facilitar el trabajo colaborativo de los actores implicados en un territorio (Bertacchini et al., 2003; Bertacchini, 2004; Dumas, 2004; Devillet et al., 2008). Y el concepto "comunicación” debe actuar primero, como hilo conductor de la información “(...) la cultura, la información, la identidad, la transmisión y el patrimonio tienen gran importancia (...) desde las ciencias de la información y la comunicación, en el proceso Datos-Información-Acción (...) se estructura la información y se gestiona, co-construyendo conocimientos y comunicaciones (...)” (Bozzano, 2013: 5); y segundo, como una "puesta en común"5 que surge cuando las comunidades se comunican y median a nivel cultural, de intercambio y de necesidades. En este sentido, hace referencia al aprendizaje colectivo ${ }^{6}$, donde los actores piensan y viven su "para qué" y la inteligencia territorial les facilita la herramienta para resolver el "cómo". Por tanto, la educación, la transmisión de conocimiento y la cultura de los actores locales, es fundamental para la construcción de las TICs y para comprender la necesidad de una intervención social.

Un debate actual e interesante, es si la inteligencia territorial es una ciencia o no. La definición empleada por la European Network of Territorial Intelligence (ENTI) cita que la "Inteligencia Territorial es la ciencia que tiene por objeto el desarrollo sostenible y que tiene por sujeto la comunidad territorial” (Girardot, 2008). Sin embargo, el mismo autor tiene una evolución al respecto, porque inicialmente plantea que es un "medio" para los investigadores (Girardot, 2000), posteriormente hace referencia a un "conocimiento multidisciplinar" (Girardot, 2002) para, finalmente, hablar de ciencia (Girardot, 2010). Pero no es el único que plantea tal controversia, Frediani (2012) también propone que la inteligencia territorial sea una forma de hacer ciencia. Sin embargo, autores como Girardot (2000), Devillet (2008)7, Masselot (2013) y Sánchez (2013) que proponen que

de las capas de chocolate de las tartas representan cada proceso y sus etapas de concreción. A su vez, la mesa se sostiene sobre un fondo que se colorea de abajo hacia arriba con distintos tonos, que representan en primer lugar a los sujetos, en segundo lugar a los objetos, en tercera posición las herramientas y finalmente los logros obtenidos.

5 Canevari, T. citado en Seminario en Minas (2013).

6 Scala, E. citado en Seminario en Minas (2013).

7 Devillet (2008) y Masselot (2013) citado en Bozzano (2013); Sánchez (2013) citado en Seminario en Minas (2013). 
la inteligencia territorial es un enfoque científico debido a su carácter multidisciplinar, no lo plantean como una ciencia. Coincidiendo con esta idea, los investigadores de esta comunicación se posicionan en el debate con la visión de que la inteligencia territorial es entendida como enfoque científico. Por tanto, se podría tomar como referencia más representativa la definición dada por Devillet (Bozzano, 2013: 5): "la inteligencia territorial es un enfoque científico colectivo de movilización de las inteligencias dentro de una región... para así, mejorar el bienestar individual y aumentar la resiliencia del territorio en el marco del desarrollo sostenible. Es un enfoque transversal que involucra la consideración de todos los sectores de actividad y la movilización de los actores del territorio e investigadores institucionales interdisciplinares...”. En lo que sí coinciden las investigaciones es en la relevancia dada al desarrollo social y medioambiental. Así, se podría pensar que ya está implícita la dimensión económica, si se tiene en cuenta que la inteligencia territorial trata de poner los recursos a disposición de los actores. Pero se puede plantear que un enfoque donde no prima el aspecto económico a priori, es complejo de aplicar, dado que la primacía del consumismo, uno de los pilares básicos del capitalismo, va en contraposición de estos territorios. Esto se ve justificado en la evolución del concepto de inteligencia territorial, donde desde un primer momento se ha intentado vincular a un concepto de origen economicista, pero que podría no corresponderse con la realidad del concepto de inteligencia territorial.

Se plantea que la "inteligencia colectiva" (IC) y la "inteligencia económica" (IE) pueden considerarse referentes conceptuales de la inteligencia territorial. De la IC se deribó la idea de "gestión del conocimiento territorial" (GCT), que trata las acciones que buscan el desarrollo humano y la mejora constante de la calidad de vida de los ciudadanos (Mora, 2003). A su vez, la IE deribó hacia la idea de "inteligencia económica territorial" (IET), entendida como la creación de productos y servicios a través de la inteligencia económica para la innovación económica de los actores y (...) el desarrollo de territorios digitales (Goria, 2006 y Gaucherand, 2006 ).

Sin embargo, y de acuerdo con Girardot (2008), estas definiciones derivadas de la IE se apartan de la dada para la inteligencia territorial. Por tanto, la IT no debe confundirse con la IET porque, si bien es cierto que ambos usan las tecnologías de la información y la comunicación como herramientas, se diferencian en cuanto a sus objetivos. La inteligencia territorial no percibe el territorio como un mercado, sino como un espacio participativo y de colaboración, pero esto ya lo plantea la IC cuando se define como "los resultados de la colaboración y el intercambio de información, así como de la competencia entre los individuos... que se puede considerar una forma de red, la cual ha sido posible gracias a la evolución reciente de la tecnología de la información" (Girardot, 2010: 29). Sin embargo, en la IET los actores y el territorio están al "servicio" de la inteligencia económica, y no al contrario, como define la inteligencia territorial, donde los actores se sirven del territorio para su bienestar y desarrollo.

8 Gaucherand, A. (2006) citado en Girardot (2008). 


\section{Gráfico 1}

\section{EVOLUCIÓN HACIA EL CONCEPTO DE IT}

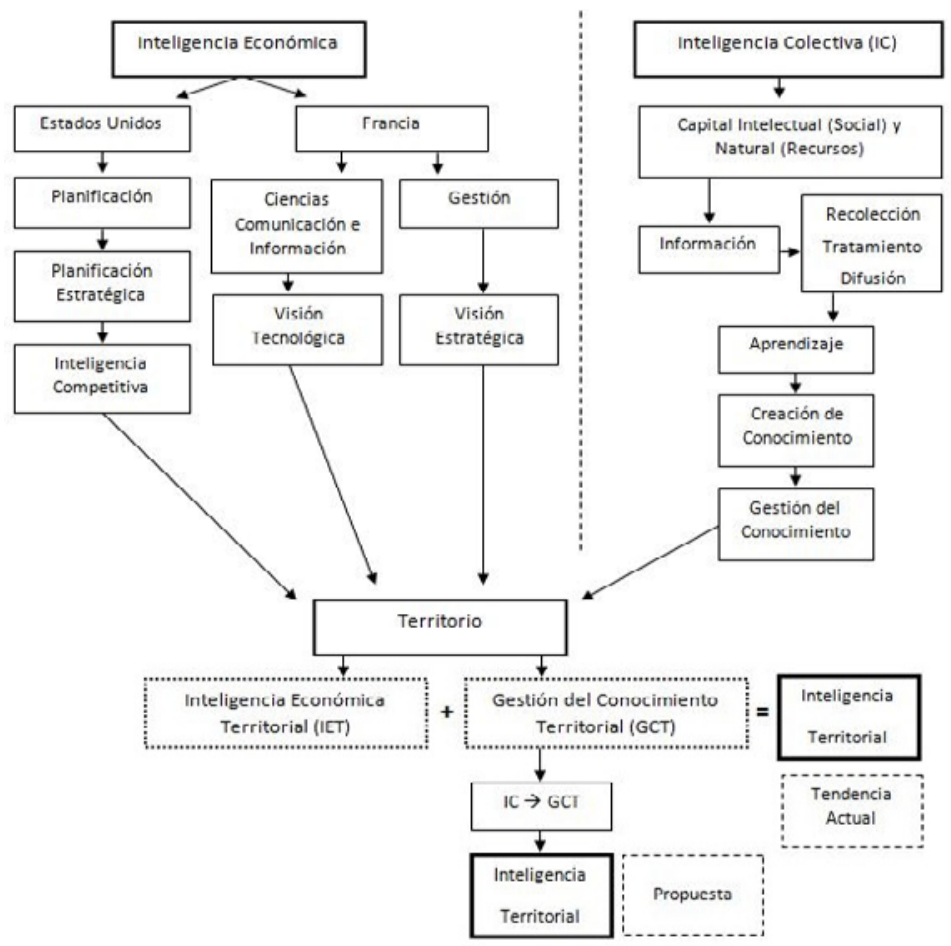

Fuente: elaboración propia.

\section{FUNDAMENTOS, HERRAMIENTAS Y APLICACIONES DE INTELIGENCIA TERRITORIAL}

\subsection{Los fundamentos de la inteligencia territorial}

Conocida la definición y el origen de la inteligencia territorial, es necesario avanzar en sus fundamentos a partir del acercamiento a sus tres elementos principales: desarrollo sostenible, gobernanza y nuevas tecnologías de la información y comunicación.

\subsubsection{Desarrollo Sostenible}

El desarrollo sostenible, entendido como una visión de futuro que es capaz de afrontar los problemas medioambientales y la distribución desigual de recursos, contiene tres dimensiones: las necesidades humanas, las limitaciones del ecosistema global y un mayor desarrollo económico. Es por ello, que el concepto está vinculado a la necesidad de una relación humano-territorio que permita la conservación del medio para que este siga siendo 
productivo. Lo que llamamos sostenibilidad es, por tanto, el resultado de un patrón de organización (De Franco, 2000). Se entiende que el desarrollo sostenible se relaciona con la calidad de vida y no debe confundirse solo con el crecimiento económico. Lo contradictorio es que a medida que crece la preocupación por la sostenibilidad, de manera implícita surge la insostenibilidad de un modelo económico dominado por la civilización industrial (Naredo, 1997), que favorece a los países del llamado primer mundo, para cada vez más empobrecer a los países con menos posibilidades pero con más recursos, que son explotados por los denominados "países desarrollados".

No obstante, el modelo de desarrollo sostenible, fundamentalmente por la ambigüedad del concepto, ha llevado a emplearlo más como una "fórmula mágica teórica" que como una aplicación real que proponga soluciones sobre el territorio, de hecho, diversos autores como Naredo (1997) plantean que bajo el paraguas del desarrollo sostenible, se han desarrollado simplemente actuaciones puramente económicas, utilizando como excusa el medio ambiente y la sociedad.

Es por estas razones, por las que la inteligencia territorial se plantea como investigación-acción. El hecho de que las comunidades humanas sean el foco principal para la inteligencia territorial, viene explicado por la importancia real que se le ha concedido al desarrollo sostenible desde un punto de vista local. Es más factible lograr un desarrollo sostenible a escala local, donde los actores están en contacto directo con su territorio. Se pretende potenciar las capacidades e iniciativas locales, la cercanía a los problemas, necesidades y recursos, así como permitir a la ciudadanía que elabore acciones y estrategias para el desarrollo. Para implementar estos modelos participativos basados en micro-problemas, micro-acciones y micro transformaciones es necesario tener en cuenta el factor escala, por la complejidad de la cooperación entre actores y de la implantación de un modelo de gobernanza.

\subsubsection{Gobernanza}

El concepto gobernanza ha sido atendido profusamente en las dos últimas décadas. En principio existe una clara diferenciación entre la idea de gobierno y gobernanza ya que, mientras que el gobierno se caracteriza por la presencia de un poder (Estado) organizado mediante los poderes públicos con relaciones jerárquicas, la gobernanza hace referencia al nacimiento de un nuevo sistema relacional más complejo, que incluye actores ajenos a la política, representando una alternativa al modelo de gestión pública (Farinós, 2008). Teniendo en cuenta las bases de la inteligencia territorial, la gobernanza debe tener un enfoque basado en redes (Messner et al., 1994; Pierre, 2000; Peters, 2006) y un sistema socio-céntrico: el foco de atención es la coordinación, el auto-gobierno y la descentralización. A su vez, se evidencia la existencia de un gobierno que pasa de ser "unidireccional" o estado-céntrico (gobernantes-gobernados) a convertirse en "bidireccional" (públicoprivado), donde la gobernanza se entiende como un fenómeno social y de colaboración para presentar soluciones y oportunidades colectivas (Kooiman, 2003).

Es necesario que las diferencias jerárquicas se minimicen y que se siga una estructura horizontal, donde los acuerdos y las decisiones sean fruto de un consenso entre los actores implicados (Torres et al., 2008). Lo "público" ha de incluir a las instituciones y a la 
sociedad, es decir, una gobernanza multinivel y de participación activa de la población. Para esto, se requiere relaciones de transferencia y coordinación (Almansa, 2010). Una de las claves del éxito es tener una base científica en las herramientas que acompañan los procedimientos de actuación, para asegurar la efectividad de una gobernanza multinivel. Una de las herramientas más utilizadas son los "observatorios territoriales", que deben ser capaces de obtener datos y rentabilizar el conocimiento generado a través de las TIC, con el fin de desarrollar procesos de planificación, gestión y evaluación, según las bases de la gobernanza.

Pero la realidad es menos esperanzadora, porque la idea de gobernanza no impera en la mayoría de gobernantes que suelen tener una visión a corto plazo y muy sesgada en relación con su ámbito de interés. Para que un territorio sea inteligente y cuente con un buen sistema de gobernanza, es necesario una cultura de cooperación no solo desde la escala local, sino a nivel regional, nacional y mundial. Donde políticos, empresarios y ciudadanos tengan similares enfoques sobre la perdurabilidad económica, social y ambiental a largo plazo, lejos de sus años de candidatura o beneficios empresariales a costa de todo. Se presenta como una ardua tarea, ya que en una economía globalizada y capitalista, no se suele ver más allá de las aspiraciones económicas y beneficios individuales.

\subsubsection{TIC}

La inteligencia territorial debería configurar y desarrollar sistemas socio-técnicos para hacer efectiva la integración social con el territorio. Para ello los actores requieren de herramientas y técnicas de comunicación, pero deben ser capaces de hacerlas evolucionar expresando las necesidades que surgen con el uso de las mismas. En este sentido, el primer requisito que se plantea la inteligencia territorial es promover la difusión de la información, en un entorno de comunicación que sea capaz de trasladar lo local a lo global, y que sirva para conocer las necesidades de una población con el fin de actuar. Desde las ciencias de la información y de la comunicación, el proceso "informacional" y antropológico supone la conjunción de tres hipótesis (Bertacchini, 2000): (1) los actores intercambian información (generación de energía a nivel individual o colectivo); (2) los actores dan credibilidad a la información que reciben (captura e intercambio de información); (3) una vez que el proceso de información se ha establecido, los actores desarrollan las redes apropiadas y transfieren sus competencias y conocimientos (movilización y transferencia de energía: formulación de un proyecto de desarrollo). La unión de estas tres hipótesis provoca que las competencias y conocimientos de los actores puedan ser identificados mediante la información que han transmitido y se pueda trasferir al resto de comunidad, así la información recabada se pueden transformar en acciones de desarrollo local.

Para que las TIC puedan proporcionar herramientas a la inteligencia territorial, se deben cumplir los siguientes requisitos (Girardot, 2010): (1) ser métodos y herramientas comprensibles para los actores y respetuosos con la ética del desarrollo sostenible; (2) dar difusión a los métodos fundamentales de análisis de los territorios (Sistema de Información Territorial) y del procesamiento de datos territoriales; (3) promover la 
aplicación de los principios de gobernanza, de intercambio de conocimiento y facilitar la investigación-acción territorial. La función de las TIC para la implementación de procesos de inteligencia territorial es básica, ya que concierne al análisis de los métodos científicos, a las herramientas genéricas como los Software y SIG, al desarrollo de una visión sistémica del concepto de territorio, a un enfoque global de la información territorial y a la implantación de los principios de colaboración y de participación.

Pero este escenario dominado por las TIC genera otros conflictos y desigualdades ya que, estas herramientas, deberían haber servido para promover la igualdad y equidad territorial, no obstante, en muchas ocasiones han servido para incrementar las diferencias y promover la decadencia de regiones, aquellas que no tienen recursos para acceder a las mismas (Calderón, 2012). Es por ello, que la inteligencia territorial plantea el diseño de herramientas adaptativas a los territorios, teniendo en cuenta las dificultades tecnológicas de los mismos y sus limitaciones. TIC que contribuyen al desarrollo, que solucionan problemas y cubren necesidades, y que sirven como medio para disminuir las desigualdades culturales, económicas y sociales existentes (Naciones Unidas, 2005).

\section{HERRAMIENTAS Y APLICACIONES DE INTELIGENCIA TERRITORIAL}

Las herramientas y métodos desarrollados desde la inteligencia territorial tienen su base en la estrecha colaboración de los actores territoriales y los equipos de investigación, enfocados en una dinámica de investigación-acción para aportar soluciones reales a las problemáticas actuales. Gran parte de las herramientas que se han desarrollado por la ENTI (Red Europea de Inteligencia Territorial) y por el equipo de investigación interdisciplinar TAG (Territorio Actores y Gobernanza para la Transformación ${ }^{9}$ ) en Latinoamérica, han sido planteadas para ser utilizadas por partenariados de actores que actúan sobre una misma problemática o espacio. Dichas herramientas se caracterizan por combinar técnicas cualitativas, cuantitativas y espaciales basadas en las TIC's. Al ser los actores partícipes de su diseño, están adaptadas a sus necesidades y son totalmente accesibles, tanto desde un punto de vista económico como por la sencillez de su uso.

En Europa Catalyse emplea un sistema de información territorial (Sitra) como principal herramienta y varios softwares (Pragma, Anaconday Nuage). Es muy común la creación de Observatorios Territoriales, aunque estos no son "nuevos operadores en el territorio, sino un espacio funcional en el que los operadores materializan los procesos de gobernanza asociados a la intervención territorial" (Almansa, 2010: 75). Estas herramientas han sido desarrolladas desde la ENTI, que reúne a los equipos de investigación y actores territoriales para diseñar, gestionar y evaluar acciones de desarrollo sostenible que mejoren el bienestar de los ciudadanos y de las comunidades territoriales.

En Latinoamérica el equipo TAG basa sus herramientas en la Perspectiva Metodológica EIDT (Entendimiento, Inteligencia y Desarrollo Territorial), cuyo objetivo es construir entre actores locales métodos de investigación e instrumentos de interven-

9 Es un equipo creado en 2007 y formado por investigadores de la Universidad Nacional de La Plata (UNLP), el CONICET (Consejo Nacional de Investigaciones Científicas y Técnicas de la Argentina) y actores asociados. TAG, a su vez, forma parte del GDRI-INTI "Groupe De Recherche International-International Network of Territorial Intelligence" (INTI). 
ción territorial que acompañen sistemáticamente a la: imagen de futuro, estrategia que llevará a ella y obtención de logros colectivos (Bozzano et al., 2010). La metodología EIDT es el fundamento que da pie a seis herramientas: (1) Observatorios de Inteligencia y Desarrollo Territorial (OIDTe) que despliega una estrategia multi-metodológica cuyas referencias son las experiencias europeas; (2) Método Territorii, empleado para analizar, interpretar y determinar un problema en ocho momentos: territorios reales, vividos, pasados, legales, pensados, posibles, concertados e inteligentes; (3) Método Stlocus, se basa en la identificación de patrones de ocupación y apropiación territorial a micro-escala; (4) Método Portulano, método cartográfico utilizado para investigaciones interdisciplinarias; (5) Método Skypa, cuyo objeto es hacer más operativa la inteligencia territorial para concretar en menos tiempo proyectos en la micro, meso y macro escala y en el corto, medio y largo plazo; (6) Método Kinético, determina que los colectivos deben estar compuestos por los actores claves de un territorio, quienes deben construir las apuestas imaginadas, negociar y acordar las formas y condiciones, y crear, transformar e innovar en las formas y maneras de logro. Una de las claves de estas herramientas es que se adaptan a cada territorio y a las características de la población. Tras analizar diversas actuaciones ${ }^{10}$ se pueden sistematizar estas experiencias según las herramientas utilizadas, los países donde se han implantado, la duración de los proyectos, la escala de trabajo y la temática abordada. Las actuaciones se han llevado a cabo principalmente en Latinoamérica $(45,6 \%)$ y en Europa $(43,8 \%)$, mientras que en el resto de continentes los trabajos disminuyen notablemente: $5,2 \%$ en África, 1,8\% en Asia y en América del Norte.

A escala nacional, destacan Argentina y España, que contabilizan el 59,6\% de todas las actuaciones. En España digno es de mencionar las actuaciones desarrolladas en la provincia de Huelva, donde se implantó el primer Observatorio de Empleo en 199611. Le siguen Francia que concentra el 7,01\%, Bélgica el 5,26\% y Uruguay el 5,26\% de las intervenciones. En Francia destaca Besançon, es el principal núcleo donde se ejecutan estas iniciativas, ya que es la cuna de Catalyse, se desarrolló el observatorio Mosaïque (1995-1999), fue la ciudad donde se llevó a cabo la primera conferencia internacional sobre inteligencia territorial a nivel mundial en 2003 y, posteriormente en 2008. Las actuaciones se organizan desde la Universidad de Franche-Comté. Además, cuenta con varios proyectos como el de la Red de los Jardínes de Cocagne y la asociación ADAPEI (Asociación para discapacitados mentales). En Bélgica, la ciudad de Lieja acoge el Observatorio Partenarial Optima e Integra +, y Uruguay centra sus actuaciones en la región de Lavalleja con proyectos turísticos. En Hungría (Pecs), Rumanía (Alba Iulia), Italia (Salerno) y Túnez (Gafsa/Menzel-Habib) han implementado dos proyectos cada una de ellas, básicamente observatorios territoriales para el desarrollo regional.

10 Se han analizado en números absolutos un total de 56 actuaciones ya que eran aquellas que contaban con información suficiente para su valoración.

11 En 2011 se amplió este observatorio con el Centro de Investigación Internacional en Inteligencia Territorial (2011); también en Huelva se encuentra la Fundación Valdocco cuyo objetivo es bienestar de la ciudadanía, la familia, la economía y la educación. 


\section{Gráfico 2 \\ NÚMERO DE APLICACIONES DE IT POR PAÍSES}

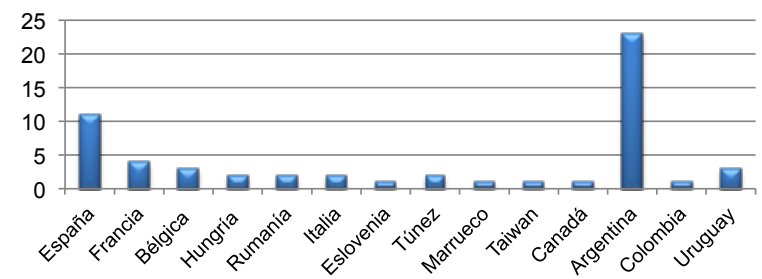

Fuente: elaboración propia.

En los países europeos (gráfico 3) han sido los Observatorios, el Método Catalyse y las herramientas Pragma y Anaconda, las herramientas más utilizadas; en Latinoamérica también destaca los OIDTe, el Método Territorii, el Stlocus, el Skypa y los talleres. En África la utilización de los SIG y Observatorios ha sido relevante, mientras que en Asia y Norteamérica han seguido el modelo europeo.

\section{Gráfico 3 \\ DISTRIBUCIÓN DEL USO DE HERRAMIENTAS DE INTELIGENCIA TERRITORIAL POR CONTINENTES}

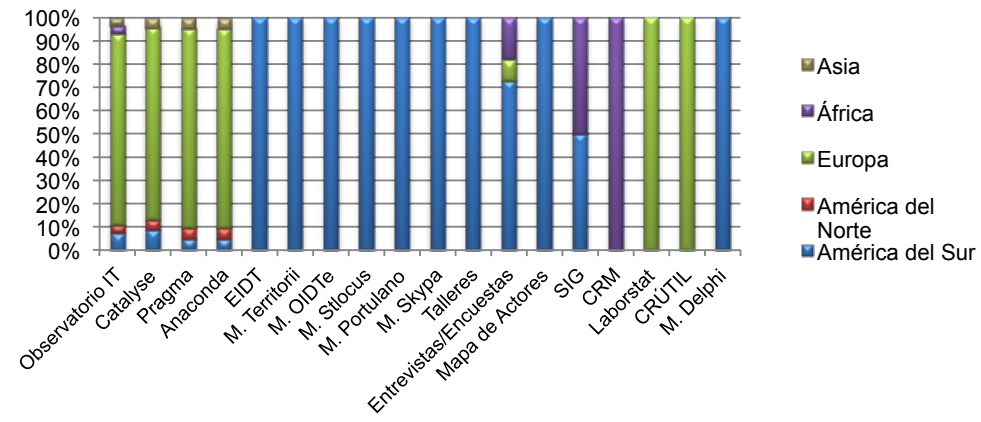

Fuente: elaboración propia.

Un rasgo a destacar es que, mientras que en Europa se plantean proyectos de base tecnológica importante, en Latinoamérica los métodos son menos tecnológicos y más humanos, es decir, hay un trabajo más directo con los actores, en forma de talleres, seminarios, etc.

Con respecto a las temáticas y proyectos abordados, en los países europeos las actuaciones has sido planes conjuntos con duración determinada, mientras que en los otros continentes, especialmente en países como Argentina y Uruguay, las intervenciones han sido más aisladas pero continuas, sin límite temporal en la mayoría de los casos. Por último, hay que resaltar que las intervenciones hasta la fecha, son a micro-escala, por tanto, de ámbito local en un $96,4 \%$ de los casos. Las temáticas afrontadas son muy diversas, agru- 
pándolas para su análisis en 19 ítems. Destaca en primer lugar, que el 70\% de las intervenciones se ha realizado en "integración de proyecto de investigación”, "inmigración”, "implantación de observatorios territoriales" y en "planificación territorial".

\section{Gráfico 4 \\ NÚMERO DE APLICACIONES DE INTELIGENCIA TERRITORIAL SEGÚN TEMÁTICA}

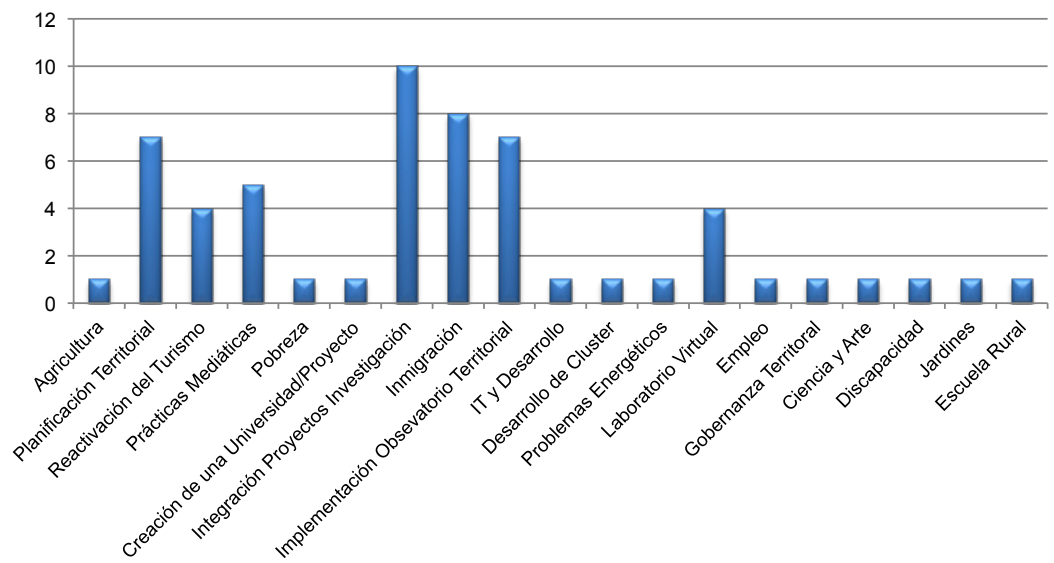

Fuente: elaboración propia.

\section{Gráfico 5}

\section{NÚMERO DE APLICACIONES DE INTELIGENCIA TERRITORIAL POR TEMÁTICA Y PAÍS}

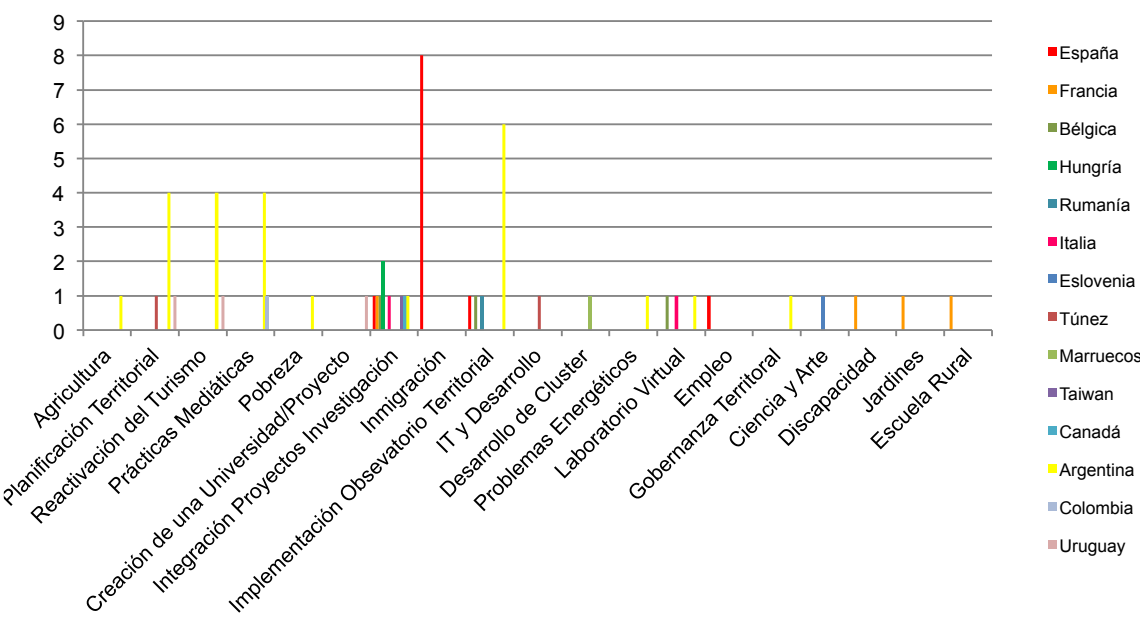

Fuente: elaboración propia. 
La "integración de proyectos de investigación" se basa en la difusión de los métodos para el análisis territorial y en el tratamiento de los datos como base para el desarrollo de una región. Se implanta básicamente en Europa, con iniciativas también en Taiwán, Argentina o Canadá. La "inmigración”, trabajado fundamentalmente en España desde la "Asociación Comisión Católica Española de Migraciones", donde se ha creado una red de siete observatorios de las migraciones. Los "observatorios territoriales" y la "planificación territorial" están muy relacionados porque se implantan generalmente de modo conjunto; parten de la base de que el territorio es un espacio "social común" y para conocer las necesidades de la población se requiere de un centro que sea capaz de captar estas necesidades y se convierte en espacio de investigación-acción.

\section{INTELIGENCIA TERRITORIAL Y TURISMO: ALGUNAS APLICACIONES}

Como se ha indicado, las temáticas abordadas con metodologías propias de la inteligencia territorial son variadas y dispares, por ejemplo, en el caso de Europa se han desarrollado actuaciones en el campo de la inmigración, los problemas sociales de las regiones desfavorecidas, el empleo, etc., mientras que en Latinoamérica, los campos de actuación han sido la agricultura, la planificación territorial, el turismo, la integración social y la pobreza.

Los proyectos de turismo han sido escasos, de hecho tan sólo se han encontrado cinco actuaciones que conjugan inteligencia territorial y turismo: (1) Uruguay (Punta del Este) en 2009, donde se trabajó en la generación de empleo, promoviendo la innovación ecológica y social, así como la puesta en valor de los recursos naturales y culturales del destino; (2) Uruguay (Lavalleja) en 2010, en la que se trató al turismo con el fin de conservar la biodiversidad y el patrimonio cultural, e investigando como repercute en la calidad de vida de la población; (3) Argentina (La Plata) en 2012, se trata de rescatar los activos patrimoniales que estén vinculados a la ciudad y a las prácticas sociales de la misma; (4) Argentina (Brandsen) en 2012, se lleva a cabo el "Parador ecológico La Posada" para revalorizar el patrimonio cultural y natural y generar vínculos con los actores locales; (5) Argentina (Santa Fé y Paraná) en 2011, se realizó el proyecto "Organización de la Inteligencia Territorial, para el Turismo Sustentable de la RMSP”.

De esta serie de iniciativas de inteligencia territorial focalizadas en el turismo, se han analizado de modo detallado las dos últimas que, al igual que las restantes, tratan de potenciarse y desarrollarse desde las propias comunidades anfitrionas (Gliemmo, 2012: 1). La forma de hacer turismo está cambiando, cada vez más se buscan experiencias y emociones, es decir, se busca al individuo de ahí que sea imprescindible que el turismo busque una valorización de las identidades locales, pero no sólo como "mito", sino como realidad, donde la estandarización y homogeneización no afecte a la cultura del mismo. La sociedad es la que crea la esencia de un lugar, la que vive el territorio y la que es anfitriona del mismo, cualquier transformación que no tenga en cuenta a los actores locales no tendrá sentido. $\mathrm{Y}$ es en este contexto, donde la intelegencia territorial aporta herramientas y experiencias para que el desarrollo del turismo pueda ser fuente de redistribución de riqueza y mejore cualquier sociedad. 


\subsection{Parador ecológico La Posada12}

El proyecto de este centro ambiental y social se realiza en el municipio de Brandsen en la Provincia de Buenos Aires, ámbito rural $\left(23 \mathrm{hab} / \mathrm{km}^{2}\right)$ en la ruta que une el Área Metropolitana de Buenos Aires y el corredor turístico del Litoral Atlántico Bonaerense (donde se ubica La Plata, uno de los destinos turísticos más importantes dentro del segmento de sol y playa del país). La propuesta de intervención nace de una problemática, la ubicación de nuevas urbanizaciones en la zonas rurales motivada por la cercanía a La Plata, lo que ha provocado una polarización social grave, con escasa integración social y territorial de estas nuevas zonas residenciales de alto poder adquisitivo, y ha deparado graves impactos ambientales por la proliferación de vertederos clandestinos que afectan a la calidad de vida de la población local.

Así, el proyecto propone un centro para la recuperación ambiental de una de las áreas degradadas por la ejecución de la Autovía 2 que une Buenos Aires con Mar del Plata. Lo importante de esta actuación no solo es el objetivo que persigue, sino el método participativo y como la información (ambiental, social y económica) ayudó a definir las necesidades. Destaca el estudio ambiental-cultural, la realización de encuestas a la población local y los talleres participativos, el proyecto se fue consensuando en cada una de sus etapas.

En definitiva, se trata de planificar de abajo-arriba, además estableciendo criterios de preservación natural, cultural y social. Esta reconversión de un espacio degradado no sólo remedia la situación medioambiental-urbanística generadora de conflictos socioeconómicos, sino que se ha creado un espacio capaz de generar apropiación (terroir ${ }^{13}$ ) por parte de la población local, donde se identifican y comparten con los visitantes.

\subsection{Organización de la Inteligencia Territorial, para el Turismo Sustentable de la RMSP14}

Esta iniciativa se organiza en torno a las ciudades de Santa Fe y Paraná (Provincia de Entre Ríos en Argentina). El proyecto surge tras detectar un contexto social problemático, donde se destacan procesos de crecimiento económico que aumenta las desigualdades sociales, bajos grados de cooperación público-privada, formación deficiente, disminución de calidad de vida por la pérdida de valor de los recursos naturales y también los culturales.

A partir de un diagnóstico participativo se establecieron dos objetivos: la creación de una Reserva de la Biosfera en Ambiente Urbano para ambas ciudades, a través de la colaboración con organización internacionales, actores académicos y políticos; y, en segundo lugar, la creación de la "Organización de la Inteligencia Territorial, para el Turismo Sustentable de la RMSP”, integrando a organizaciones institucionales en materia

12 Actor dinamizador: Universidad Nacional de La Plata - Facultad de Arquitectura y Urbanismo- Facultad de Ciencias Económicas. IIPAC - Instituto de Investigaciones y Políticas del Ambiente Construido.

13 El término "Terroir", es el que delimita una determinada zona geográfica la cual queda definida por una comunidad, donde esta es la que construye su historia, donde prima el conocimiento colectivo y se basan en prácticas que integran el entorno natural y el factor humano (ORMAUX, 2010). Es un área creativa, innovadora y que se fundamenta en la tradición. Trata de identidad de la población hacia "su" territorio, tomando el espacio donde habitan como una propiedad por derecho y donde tiene lugar la idiosincrasia de ese pueblo.

14 Actor dinamizador: REDES (Asociación "Red Entrerriana para el Desarrollo Sostenible"). 
política, científica, tecnológica y de formación, asociaciones empresarias y la ciudadanía. El turismo se planteó como elemento clave dado el crecimiento en ambas ciudades con tres acciones: (1) estrategias, programas y proyectos turísticos donde la sostenibilidad era la referencia, (2) se creó una red de cooperación con representación de todos los actores, (3) y se planificó la creación de un Centro Regional de Inteligencia Territorial para el Desarrollo Sostenible, a modo de observatorio.

En conclusión, ambos casos difieren por la naturaleza de los proyectos, el tipo de espacio, el grado de desarrollo turístico, etc., sin embargo, la clave de ambos es el método y los objetivos para la resolución de conflictos. Se inician con la co-construcción en su primera etapa de diagnóstico, donde los problemas son trasmitidos también por los actores, y sigue la co-participación para proponer acciones de mejora; es la transformación de abajo hacia arriba, ahondando en las personas y en su implicación, lo que conlleva una apropiación de las iniciativas por tomarlas como suyas. Es ahora donde se comprende lo que Bozzano (2011) identifica como el triple objetivo simultáneo que debe aportar la inteligencia territorial al turismo: sujetos, proyectos y territorios, y se ha de trabajar por identidades, necesidades y expectativas.

\section{UNA ÚLTIMA REFLEXIÓN}

Esta comunicación nace de una línea de investigación conjunta, planteada desde la Facultad de Turismo de la Universidad de Málaga, donde tres grupos de investigación (Análisis Geográfico Regional, Turismo y Territorio, Grupo de Sistemas de Información Cooperativos) trabajan de modo integrado los procesos que vinculan la actividad turística, los territorios y el concepto de "inteligencia". Se prevé que en los próximos años, la relación entre inteligencia territorial y turismo, sea una temática de enorme interés, bien desde el nuevo concepto "Destinos Turísticos Inteligentes" (DTI), bien desde la Inteligencia Territorial aplicada a los Destinos Turísticos.

En estas líneas, se ha apostado por una lectura de los procesos de "inteligencia" aplicados a los territorios turísticos centrada en la gobernanza y el trabajo con los actores, más que en un enfoque sólo y exclusivamente tecnológico, que es aquel por el que están apostando los nuevos "destinos turísticos inteligentes" olvidándose, en la mayoría de los casos, de los actores del territorio, verdaderos orquestadores en cualquier proceso de cambio y mejora. Se trata de que las tecnologías sean los hilos conductores de las informaciones y el conocimiento, pero no sean el fin de las actuaciones. Es aquí donde surge el punto de inflexión, en el uso de las TICs, ya que es este matiz el que fundamenta la principal diferencia entre los "Destinos Turísticos Inteligentes" y la "Inteligencia Territorial aplicada a los Destinos Turísticos".

En este momento, donde la valorización de diferentes espacios por parte del turismo tiende a reproducir atractivos, muchas veces desconectados de su historia e identidades, de sus características ambientales y desarticuladas de otras actividades económicas, se plantea que la inteligencia territorial aporta una base teórico-metodológica robusta y contrastada. La Inteligencia Territorial puede ser aplicada a cualquier destino turístico, sea cual sea el grado de madurez, el segmento turístico, etc., ayudando a mejorar los procesos de planificación y gestión del turismo y del destino. De esta manera, se ten- 
dría una visión más holística y consensuada que repercutiría positivamente en todos los actores implicados. Para ello, los planificadores y gestores de los destinos deben anticiparse y adaptarse a las nuevas necesidades del turismo, y el concepto y las herramientas de inteligencia territorial pueden ayudar al nuevo paradigma emergente del sector turístico. En este nuevo paradigma los turistas se movilizan cada vez más en busca de la diversidad sociocultural, de nuevas prácticas que evocan a la experiencia, al regreso del individuo y la ruptura de la vida cotidiana; a su vez, requiere de una mayor preservación del medio -en su sentido más amplio-, se refiere a mantener los valores de una sociedad y su identidad, que no sea un territorio "teatralizado" por el turismo, y que sea el desarrollo de la actividad turística un medio para mejorar el tejido social, económico y ambiental.

Cabe plantear que la inteligencia territorial es capaz de abarcar las problemáticas expuestas en esta investigación -delimitación territorial y social, sostenibilidad y gobernanza-, pudiendo presentarse como una nueva alternativa a los modelos tradicionales de planificación y gestión del turismo. Donde la participación de todos los actores es vital para planificar y gestionar, ya que a través de las herramientas que aporta la inteligencia territorial es posible llevar a cabo esta participación activa.

No obstante, la principal dificultad que se plantea para adaptar el marco teóricometodológico de la inteligencia territorial a cualquier destino turístico, es la escala. Hasta hoy, las intervenciones que se han realizado han sido a micro-escala, y un destino turístico, por norma general, es un territorio más amplio y complejo. Una forma de solventar dicho inconveniente sería a través de intervenciones en los distintos "distritos" (Dredge, 1999) que conforman el destino turístico, dado que la suma de los diferentes "distritos", da lugar a "condados" que conforman el destino. A modo de ejemplo: la Costa del Sol supondría un destino turístico, Málaga Capital un "condado" y el centro histórico de Málaga un "distrito".

El ejemplo de área de estudio no ha sido aleatorio, este estado de la cuestión y los problemas de escalas son el inicio de una línea de investigación futura, donde se plantea utilizar las herramientas propias de la inteligencia territorial en los procesos de gestión y planificación de los destinos turísticos, tomando como área de intervención y experimentación el centro histórico de la ciudad de Málaga. El fin de dicha línea de investigación es determinar en qué medida y de qué forma inciden las prácticas turísticas en la co-construcción de un territorio turístico, entendido desde la identidad, necesidad y expectativas de los actores locales.

En definitiva, proponer acciones de inteligencia territorial en los destinos turísticos de la mano de la investigación-acción puede ser positivo, si se avanza en la gobernanza, entendida como el buen gobierno transparente y participativo, y en la sostenibilidad, si el turismo sirve para mejorar el "estado de justicia" como plantea la filósofa Adela Cortina. Esta catedrática de Ética de la Universidad de Valencia entiende que lo justo es cubrir las necesidades básicas de alimentación, educación, sanidad, etc. (Rodríguez, 2014) a lo que habrá que añadir un medio ambiente saludable y perdurable, con todo lo que ello conlleva en nuestro actual modelo económico capitalista con tendencia neoliberal. En conclusión, si la inteligencia territorial solo avanza en tecnología, pese a lo positivo que resulta una mayor eficiencia energética y una movilidad más sostenible, los avances 
“inteligentes" serán muy parciales; parafraseando al arquitecto y filósofo Eduardo Prieto (2014) “La ciudad es el problema; la técnica, la solución”, ¿es el territorio el problema? ¿es el destino el problema?, parece ser que no, de ahí que la relación entre inteligencia territorial y destinos turísticos suponga una línea de investigación presente y futura de enorme interés.

\section{BIBLIOGRAFÍA}

ALMANSA, B. (2010): «Inteligencia territorial para una redefinición eficiente de las políticas públicas», Revista andaluza de relaciones laborales, $n^{\circ} 23$, pp. 75-94.

BERTACCHINI, Y. (2000): «How to federate some local resources by developing new links?», ISA Research Committee, vol. 23.

BERTACCHINI, Y. (2000): Information et Veille Territoriales: Représentation du complexe local et émergence d'un projet d'intelligence territoriale. Thèse de Doctorat en Sciences de l'information et de la communication, Université d'Aix-Marseille III.

BERTACCHINI, Y. (2004): «Entre information \& processus de communication: l'intelligence territoriale», Revue Humanisme et Entreprise, n 267, pp. 244-245.

BERTACCHINI, Y. (2012): «Between Information and Communication Process, the Territorial Intelligence, as a Network Concept \& a Framework to Shape Local Development», International Journal of Humanities and Social Science, $\mathrm{n}^{\mathrm{o}}$ 18, pp. 242-247.

BERTACCHINI, Y. y HERBAUX, P. (2003): «Mutualisation \& Intelligence Territoriale», ISMD, $\mathrm{n}^{\circ}$ 14, pp. 103-113. Disponible en: http://isdm.univ-tln.fr/PDF/isdm14/ isdm14.pdf

BOZZANO, H. (2009): «Territorios: El Método Territorii. Una mirada territorial a proyectos e investigaciones no siempre territoriales», 8th International Conference of Territorial Intelligence, November 4th - 7th 2009, Salerno, Italia, pp.10, INTI-International Network of Territorial Intelligence. Disponible en: https://halshs.archivesouvertes.fr/halshs-00533337/document

BOZZANO, H. (2010): «Transformación entre actores políticos y actores territoriales con sustento científico», V Congreso Internacional Crisis de la globalización económica y el crecimiento insustentable en América Latina, octubre de 2010, Guayana, Venezuela. UNEG.

BOZZANO, H. (2011): «Inteligencia Territorial: Criterios para la concreción de proyectos». Jornadas sobre políticas públicas para el desarrollo estratégico, San Carlos de Bariloche. Disponible en https://www.slideshare.net/equipotag/inteligencia-territorialcriterios-para-la-concrecin-de-proyectos

BOZZANO, H. (2013): «Geografía e Inteligencia Territorial. Geo-grafein, Geo-explanans, Geo-transformare», Revista Geográfica Digital. IGUNNE, nº 19, pp. 1-24.

BOZZANO, H. (2013): «Iniciativas y métodos. Inteligencia Territorial, ciencia con y para la gente». A pesquisa cientifica e a cooperacao para uma sociedade mais justa, $12 \mathrm{de}$ septiembre de 2013. Unversidad do Este do Estado do Paraná. Paraná, Brasil. Disponible en: http://portalpos.unioeste.br/media/File/desenvolvimento_rural_sustentavel/ Conferencia-Exp_Desenv_Territ_em\%20confronto.pdf 
BOZZANO, H., BARRIONUEVO, C. y CIRIO, G. (2010): «OIDTe: Los Observatorios de Inteligencia y Desarrollo Territorial. Casos en Entre Ríos, Argentina», V Congreso Internacional Crisis de la globalización económica y el crecimiento insustentable en América Latina, octubre de 2010, Guayana, Venezuela, pp. 49-64, UNEG. Disponible en http://www.lavalleja.gub.uy/c/document_library/get_file?folderId=145217\&name= DLFE-3744.pdf

CALDERÓN, F. (2012): La protesta social en América Latina. Cuaderno de Prospectiva Política I. Buenos Aires, Siglo Veintiuno Editores.

DE FRANCO, A. (2000): «¿Por qué Precisamos de un Desarrollo Local Integrado y Sostenible?», Instituciones y Desarrollo, n 6, pp. 133-168.

DEVILLET, G. y BREUER, C. (2008): «Contribution to the applied territorial intelligence: reasoned catalog of territorial information available on internet and sources in Europe», VI International Conference of Territorial Intelligence, octubre 2008, Besançon, Francia, MSHE. Disponible en: https://halshs.archives-ouvertes.fr/halshs-00487197

DREDGE, D. (1999): «Destination place planning and design», Annals of Tourism Research, $\mathrm{n}^{\circ} 4$, pp. 772-791.

DUMAS, P. (2004): «Intelligence, Territoire, Décentralisation ou la Région à la française», ISDM, $\mathrm{n}^{\circ} 16$, pp. 92-95. Disponible en: http://isdm.univ-tln.fr/PDF/isdm16/isdm16.pdf

FARINÓS, J. (2008): «Gobernanza territorial para el desarrollo sostenible: estado de la cuestión y agenda». Boletín de la Asociación de Geógrafos Españoles, vol. 46, pp. 11-32.

FREDIANI, J. (2012): «Inteligencia Territorial y Transformación II. El lugar del Estado. Aplicación a cinco casos». En Experiencias Innovadoras en Investigación Aplicada. San Salvador de Jujuy, Ediciones DASS-UCSE, pp. 475-495.

GIRARDOT, J. (2000): «Principes, Méthodes et Outils d`Intelligence Territoriale. Évaluation participative et Observation Coopérative», Séminaire européen Conhecer melhor para agir melhor, May 2000, Evora, Portugal, pp. 7-17. Disponible en: https://halshs. archives-ouvertes.fr/halshs-00539811

GIRARDOT, J. (2002): «L〉intelligence Territoriale», Mélanges Jean-Claude WIEBER, Annales Littéraires de Franche-Comté, Besaçon, pp. 10.

GIRARDOT, J. (2008): «Evolution of the concept of territorial intelligence within the coordination action of the European network of territorial intelligence», Res-Ricerca e Sviluppo per le politiche sociali, pp. 11-29.

GIRARDOT, J. (2009): «Inteligencia Territorial y Transición Socio-Ecológica», Revista andaluza de relaciones laborales, $\mathrm{n}^{\circ} 23$, pp. 15-39.

GIRARDOT, J. (2010): «Qu>est-ce que l>intelligence territoriale», Savigny-Avenir, $\mathrm{n}^{\circ} 73$. Disponible en: http://www.savigny-avenir.fr/2014/01/06/quest-ce-que-lintelligenceterritoriale-jean-jacques-girardot/

GIRARDOT, J. (2013): «Le concept d'intelligence territoriale, domaines et réseaux». En Intelligence et gouvernance territoriales. Université de Franche-Comté, pp. 31-70.

GLIEMMO, F. (2012): «Inteligencia Territorial y Turismo: un diálogo hacia la definición de Lugares. Necesidades y expectativas. Estudios de casos en Argentina y Uruguay», 11th Annual International Conference of Territorial intelligence of INTI Territorial 
intelligence and globalization tensions, transition and transformation, octubre de 2012, La Plata, Argentina, pp. 1-12. Disponible en: https://halshs.archives-ouvertes.fr/ halshs-00941343/document

GORIA, S. (2006): L'Expression du problème dans la Recherche d'Informations: Application à un contexte d'Intermédiation Territoriale. Ecole doctorale Langages, Université Nancy II. Disponible en: https://tel.archives-ouvertes.fr/tel-00011918

KOOIMAN, J. (2003): «Governing as Governance», International Public Management Journal , n⿳ 3, pp. 439-442.

MASSELOT, C. (2004): Accessibilité et qualité des systèmes d'information multimédia: transfert méthodologique et technologique. Doctorat SIC, Université de FrancheComté.

MASSELOT, C. (2008): «Territorial Intelligence Communicational and Community System (TICCS)». Res-Ricerca e Sviluppo per le politiche sociali, vol 1-2, pp. 90-104.

MIEDES, B. y FERNÁNDEZ I.M. (2010): «Inteligencia territorial para la lucha contra la pobreza:aprendizajes de 20 años sobre el terreno». Revista Andaluza de Relaciones Laborales, $\mathrm{n}^{\circ} 23$, pp. 41-73.

MORA, J. (2003): «Gestión Territorial del Conocimiento (GTC) y desarrollo rural en América Latina y el Caribe. La reconstrucción de las instituciones rurales en el ámbito de los servicios de asistencia técnica», Antigua, pp. 23-26.

NACIONES UNIDAS (2005): Objetivos del desarrollo del milenio: una mirada desde América Latina y el Caribe. Santiago de Chile, CEPAL. Disponible en: https://www. cepal.org/es/publicaciones/2797-objetivos-desarrollo-milenio-mirada-america-latinacaribe

NAREDO, J. (1997): «Sobre el oriegen, el uso y el contenido del término sostenible», Cuadernos de Investigación Urbanística, $\mathrm{n}^{\circ}$ 41, pp. 7-18.

ORMAUX, S. (2010): «Products of terroir, between environment, culture and territorial development», 8th International Conference of Territorial Intelligence, November 4th - 7th 2009, Salerno, Italia, pp.7, 2010, INTI-International Network of Territorial Intelligence. Disponible en: https://halshs.archives-ouvertes.fr/halshs-00534672

PETERS, B. (2006): «Globalización, gobernanza y Estado: algunas proposiones acerca del proceso de gobernar», XI Congreso Internacional del CLAD sobre la Reforma del Estado y de la Administración Pública, Ciudad de Guatemala, pp. 103-115.

PIERRE, J. (2000): Debating Governance: Authority, Steering, and Democracy. New York, Oxford University Press.

SÁNCHEZ, N., BETANCOURT, M. y FALCÓN, M. (2012): «Acercamiento teórico al desarrollo local sostenible y su repercusión para el turismo», El Periplo Sustentable, $\mathrm{n}^{\mathrm{o}} 22$, pp. 7-36.

SEMINARIO EN MINAS (2013): ¿Qué es la Inteligencia Territorial? Por integrantes de la INTI-Miradas Complementarias. Formación en Inteligencia Territorial Uruguay 2012-2017. Minas, Observatorio de Inteligencia y Desarrollo Territorial. Disponible en: http://www.lavalleja.gub.uy/c/document_library/get_file?folderId=28672\&name= DLFE-7303.pdf

SOULIER, E., NEFFATI, H., LEGRAND, J., ROUSSEAU, F., BUGEAUD, F., CALVEZ, P. y SAUREL, P. (2011): «Territorial assemblages simulation for territorial intelligence», 
10th International Conference of Territorial Intelligence, septiembre de 2011, Liège, Belgium, pp. 9. Disponible en: https://halshs.archives-ouvertes.fr/halshs-00777450/ file/LiA_ge_2011_Soulier_Territorial_assemblages_simulation_for_territorial.pdf

TORRES, G. y RAMOS, H.A. (2008): «Gobernanza y territorios: notas para la implementación de políticas para el desarrollo», Revista Mexicana de Ciencias Políticas y Sociales, $\mathrm{n}^{\circ}$ 203, pp. 75-96. 